\title{
REDES DE DEFENSA TRANSNACIONALES \\ FRENTE A ESTRUCTURAS POLÍTICAS \\ FEDERALES. EL CASO AFROMEXICANO
}

Bianca Silvia Ramirez Navarro(a) y Claire Wright ${ }^{(b)}$

TRANSNATIONAL DEFENSE NETWORKS IN

FEDERAL POLITICAL STRUCTURES.

THE AFRO-MEXICAN CASE

REDES TRANSNACIONAIS DE DEFESA DIANTE DE

ESTRUTURAS POLÍTICAS FEDERAIS.

O CASO AFRO-MEXICANO

Fecha de recepción: 15 de febrero del 2018

Fecha de aprobación: 2 de noviembre del 2018

Disponible en línea: 23 de enero del 2019

\section{Sugerencia de citación:}

Ramírez Navarro, B. S. y Wright, C. (2019). Redes de defensa transnacionales frente a estructuras políticas federales. El caso afromexicano. Razón Crítica, 6, 257-288, doi: 10.21789/25007807.1424

(a) Bianca Silvia Ramírez Navarro

Licenciada en Estudios Internacionales de la Universidad de Monterrey

Profesora de la Universidad de Monterrey, México

https://orcid.org/0000-0003-4378-2949

bianca.ramirez@udem.edu

(b) Claire Wright

Licenciada en lenguas modernas (Filología Francesa e Hispánica) de la Universidad de Oxford, Maestría en Estudios Latinoamericanos del Instituto Interuniversitario de Iberoamérica y

Portugal de la Universidad de Salamanca y Doctorado en Procesos Políticos Contemporáneos de la Universidad de Salamanca, España

Profesora de la Universidad de Monterrey, México

https://orcid.org/0000-0002-7789-8838

claire.grace@udem.edu 


\section{R E S U M E N}

En este artículo se pretende realizar dos aportes: por una parte, brindar elementos teóricos a los estudios sobre el activismo transnacional, y, por otra con, elementos empíricos al estudio del proceso de reconocimiento de la población afrodescendiente en el caso mexicano. Tomando en cuenta la Encuesta Intercensal 2015 del Instituto Nacional de Estadística y Geografía (INEGI), alrededor de 1,4\% de la población de México es afrodescendiente; allí se observa cómo este sector se ha organizado a lo largo de los años para reivindicar su reconocimiento constitucional. A través de la creación de una red de defensa que comienza a fortalecerse tanto en el contexto doméstico como en el internacional en los últimos años, el movimiento afromexicano ha logrado tener reconocimiento político gracias a una estructura de oportunidades política internacional abierta y una política nacional que en el ámbito federal ha sido cerrada, mientras que en lo estatal ha mostrado mayor apertura. La incidencia aquí mencionada se logra con el apoyo de actores internacionales o la influencia de eventos internacionales como el año y el decenio de los afrodescendientes, así como la Conferencia de Durban.

PALABRAS CLAVE: redes de defensa transnacionales, reconocimiento político, derechos humanos, afrodescendientes, movimiento afromexicano 


\section{A B S T R A C T}

This article is aimed at making two main contributions: first, to provide theoretical elements to the study of transnational activism, and, second, to introduce empirical elements to the study of the recognition process of the Afro-descendant population in the Mexico. According to information from Inegi's Intercensal Survey 2015, around 1.4\% of Mexican population of Mexico is of African descent, showing how these population has been organizing over the years to claim constitutional recognition. Through the creation of a defense network that has been strengthening both in the domestic and the international contexts during recent years, the Afro-Mexican movement has achieved political recognition thanks to an open international policy structure of opportunities that at the federal level is mainly closed; showing greater openness at State level. The impact of the actions described in this work is achieved with the support of international actors or the influence of international events such as the year and the decade of the Afro-descendants, as well as the Durban Conference.

KEY WORDS: transnational defense networks, political recognition, Human Rights, Afro-descendants, Afro-Mexican

movement

\section{R E S U M O}

Este artigo tem como objetivo realizar duas contribuições: em primeiro lugar, proporcionar elementos teóricos aos estudos sobre o ativismo transnacional e, em segundo lugar, elementos empíricos ao estudo do processo de reconhecimento da população afrodescendente no caso mexicano. Tendo em conta o Censo 2015 do Inegi (Instituto Nacional de Estatística e Geografia), cerca de 1,4\% da população do México é afrodescendente. Além disso, também é possível observar como esse setor foi organizado ao longo dos anos para reivindicar seu reconhecimento constitucional. Através da criação de uma rede de defesa que começa a se fortalecer nos últimos anos, tanto no contexto nacional quanto internacionalmente, o movimento afro-mexicano teve reconhecimento político graças a uma estrutura de oportunidades política internacional aberta e uma política nacional que, no âmbito federal, foi fechada, enquanto o estado tem mostrado maior abertura. A incidência aqui mencionada é obtida com o apoio de atores internacionais ou a influência de eventos internacionais, como o ano e a década dos afrodescendentes, bem como a Conferência de Durban.

PALAVRAS-CHAVE: afro-descendentes, direitos humanos, movimento afro-mexicano, reconhecimento político, redes transnacionais de defesa. 


\section{N T R O D U C C I Ó N}

Las redes de defensa transnacionales centradas en la justicia social se convirtieron en actores principales de la política mundial en la década de 1990, transfiriendo ideas y recursos a sus afiliados locales y aumentando la presión sobre los gobiernos nacionales para luchar contra los abusos de los derechos humanos y la desigualdad (Htun, 2004). Existen casos paradigmáticos de movimientos afrodescendientes en América Latina. Rojas (2013) señala que, por cuestiones históricas, así como el gran volumen de población negra en Brasil y Colombia, sus respectivos movimientos afrodescendientes han logrado tener un mayor impacto político. En ambos casos los procesos de reconocimiento constitucional de esta población fueron liderados en mayor parte por activistas, además de que las redes transnacionales de defensa jugaron un papel clave (Rojas, 2013). En este contexto, desarrollos internacionales como la Conferencia de Durban, el decenio internacional de los afrodescendientes, que el Gobierno mexicano subscribió, así como diversos tratados mundiales sobre derechos humanos y antirracismo, contribuyen a la difusión de normas sobre el comportamiento apropiado que deben tener los estados liberales y democráticos, lo cual a su vez ayuda a transformar los intereses nacionales. Estos factores también permiten fortalecer a los actores políticos nacionales y aumentan la relevancia de ciertas ideas basadas en principios.

En este contexto, llama la atención la ausencia de México de la lista de estados que han reconocido a los pueblos y personas afrodescendientes en su Constitución -Brasil, Bolivia, Colombia, Ecuador y Nicaragua (Quintana \& Alanís, 2017)—. Si bien la población que se autoidentifica como afrodescendiente representa una fracción de la población mexicana 
(un poco más del 1\%), se trata de más de un millón de personas y en los últimos años diversas organizaciones afromexicanas han ganado visibilidad en la esfera pública en lo local, nacional e incluso internacional (PeñalozaPérez, 2017). Tal visibilidad también se ha dado en lo académico, en parte debido a vínculos entre activistas y académicos, así como una serie de estudios que — sobre todo desde la década de 1990 — empieza a interesarse en la situación tanto social como política de los afrodescendientes en México (Velásquez \& Iturralde, 2016). En este contexto y teniendo en cuenta otras experiencias en la región, cabe preguntarse cómo se ha dado el activismo afromexicano en México y cómo se ha vinculado con las redes de defensa transnacionales.

Este estudio busca ofrecer dos aportes: por una parte, proporcionar elementos teóricos a los estudios sobre el activismo transnacional, y, por otra, brindar elementos empíricos al estudio del proceso de reconocimiento de la población afrodescendiente en el caso mexicano. Concretamente, el objetivo del presente estudio es analizar las interacciones del movimiento afromexicano con una red transnacional de defensa de sus derechos, así como los impactos que han tenido estas interacciones en el ámbito nacional y local. Para guiar el análisis se parte de la propuesta de estructuras de oportunidades políticas articulada por Martí y Silva (2014), si bien - debido a la estructura federal del sistema político mexicano- en este artículo el espacio doméstico se divide entre lo federal y lo estatal. En términos metodológicos, se trata de un estudio de caso a base de trabajo de campo realizado en México, así como la revisión de informes, notas de prensa y otra documentación secundaria.

El artículo comienza con la mención de las bases teóricas y conceptuales que formarán la base del análisis, incluyendo el constructivismo, la identidad, las redes de defensa transnacionales y las estructuras de oportunidades políticas; después, se ofrecerá una breve reseña del pueblo afromexicano, así como la emergencia del movimiento; luego se pasa al estudio de caso, que, primeramente, analiza la influencia de las redes de defensa transnacionales en la búsqueda del reconocimiento político de los afromexicanos en el contexto federal, para luego examinar el mismo proceso en lo local, en tres estados clave: Oaxaca, Guerrero y Ciudad de México. Finalmente, se ofrecen las conclusiones del estudio, resumiendo los hallazgos empíricos y teóricos. 


\section{Consideraciones teóricas}

Al abordar la identidad del movimiento afromexicano y su relación con actores internacionales, el principal enfoque teórico para el análisis es el constructivismo. A diferencia de otras corrientes teóricas de las relaciones internacionales, cuyos fundamentos teóricos son de corte economicista, el constructivismo tiene una base sociológica (Anaya, 2014, pp. 32-33).

Para Wendt (el autor más representativo de esta corriente), la identidad es un concepto clave dentro del constructivismo (Zehfuss, 2009, p. 15). Por lo mismo, antes de continuar, conviene mencionar que, de acuerdo con Bello (2004), la identidad de un individuo se construye a partir de un conjunto de condiciones y momentos. A su vez, estas condiciones y momentos influyen en el autoconcepto que tiene un individuo, "[...] que se deriva del conocimiento de su pertenencia a un grupo o grupos sociales juntamente con el significado valorativo y emocional asociado a esta pertenencia”, lo que constituye así la identidad social (Tajfel, como se cita en Valera y Pol, 1994, p. 8).

Las identidades sociales implican una identificación con el destino del otro. Así, Wendt sostiene que la forma en que se lleva a cabo la política internacional se crea a través de una construcción social, porque las identidades y los intereses se construyen y respaldan por la práctica intersubjetiva. Las nociones tanto de uno mismo como del entorno dan forma a las interacciones y se forman por las interacciones. De este modo, se crea la realidad social (Zehfuss, 2009, p. 12). De acuerdo con Wendt (1992), los significados colectivos constituyen las estructuras que organizan nuestras acciones. Los actores adquieren identidades al participar en estos significados colectivos (Wendt, 1992, p. 397). La relevancia de las identidades puede variar, pero cada una es una definición inherentemente social del actor que se basa en las construcciones que los actores colectivamente tienen sobre sí mismos y entre sí, y que constituyen la estructura del mundo social. De la misma forma, las identidades son la base de los intereses (Wendt, 1992, p. 398). Así como cada persona tiene diversas identidades vinculadas a papeles institucionales, un Estado puede tener múltiples identidades (Wendt, 1992, p. 398). Una identidad se puede construir alrededor de una serie de valores o principios que definen 'el ser' de un Estado (Anaya, 2014, p. 33).

Para fines del caso, un concepto que se define dentro de la identidad es la etnicidad. "[...] trata del origen en una geografía cultural en la 
cual una persona absorbe la cultura de un lugar (casi 'en la sangre') de las generaciones anteriores" (Wade, 2000, pp. 29-30). La Comisión Económica para América Latina y el Caribe (Cepal) (2000) vincula la etnicidad a factores de orden cultural que integran la identidad étnica. El constructivismo y el relativismo cultural, para los cuales tanto la raza como factores étnicos son construcciones sociales y culturales, inciden en esta identidad.

Regresando al plano internacional, según Anaya (2014, p. 33): “[p] ara el constructivismo, el sistema internacional es un 'contexto social', el cual es definido, precisamente, por el marco internacional de identidades y normas existente". Dentro de este contexto conviven, además del Estado, otros actores, denominados por Anaya (2014) como "emprendedores de normas". Este enfoque considera al Estado como el actor más relevante en el sistema internacional; sin embargo, a veces es necesario el activismo de emprendedores de normas para que la lógica de lo apropiado se ponga en funcionamiento (Anaya, 2014, p. 34). Estos emprendedores que incursionan como actores centrales dentro del sistema internacional pueden ser individuos, grupos o instituciones (gubernamentales, no gubernamentales e intergubernamentales) que proponen nuevas normas e identidades "[...] o que utilizan las ya existentes para presionar o persuadir a los Estados a actuar de manera consecuente con las identidades que dicen tener y con las normas aceptadas por el grupo al que pretenden pertenecer" (Anaya, 2014, p. 35).

Recientemente, los estudios constructivistas se han enfocado en el papel de los actores no estatales antes mencionados en la política mundial, junto con las ideas que los actores difunden y cómo esas ideas pueden transformar la realidad social internacional. Estos actores forman parte de un nuevo ámbito el cual corresponde al estudio del activismo transnacional (como es el caso los movimientos afrodescendientes en América Latina) y del en el cual se profundizará ahora (De la Torre, 2011, p. 54).

En ocasiones, para lograr mayor incidencia en el contexto nacional, los activistas domésticos se internacionalizan. De acuerdo con Tarrow (2005), una de las características más importantes del activismo es cómo se conecta en el ámbito doméstico y global construyendo un proceso transnacional. A través del activismo transnacional, colectivos sociales han logrado influenciar en distintos grados la toma de decisiones por parte de los gobiernos en su política interna y externa, principalmente en materia de derechos humanos (Anaya, 2014, p. 118). 
Tarrow (2005) sostiene que el internacionalismo de los activistas sociales forma parte de una estructura triangular y densa de las relaciones entre los estados, los actores no estatales y las instituciones internacionales, y, a su vez, esta estructura produce las oportunidades para que los actores participen en acciones colectivas en los diferentes niveles de este sistema. Si bien el internacionalismo va más allá de las instituciones, los regímenes y los procesos internacionales concretos, estos se encuentran en su núcleo y proporcionan una estructura de oportunidades y amenazas para los actores no estatales (Tarrow, 2005, p. 25).

Antes del texto de Tarrow, Keck y Sikkink (1998) ya habían definido el concepto de redes de defensa transnacionales, formadas por principios compartidos de ideas y valores que en conjunto forman una identidad colectiva, la cual, de acuerdo al constructivismo, está basada en sentimientos de solidaridad, comunidad y lealtad (Zehfuss, 2009, p. 15). A su vez, estas redes suelen ser significativamente transnacionales, regionales y domésticas. Como se observa en la teoría constructivista, estos nuevos actores contribuyen a una convergencia de normas sociales y culturales capaces de dar soporte a procesos de integración tanto regional como internacional (Keck \& Sikkink, 1998).

Las redes de defensa transnacionales generalmente están conformadas por diferentes emprendedores de normas, entre los cuales se destacan grupos de la sociedad civil —oNG nacionales e internacionales-, los cuales propician el surgimiento de estas redes, los órganos de regímenes internacionales de derechos humanos así como agencias y los funcionarios de gobiernos democráticos. Estos actores forman parte de un esquema horizontal e informal, en el cual intercambian información y servicios para promover agendas que se basan en principios y que, en gran medida, alimentan y mantienen su dinamismo (Anaya, 2014, pp. 118-119).

Las redes funcionan mejor cuando son densas, con muchos actores, fuertes conexiones entre los grupos de la red y flujos de información confiables. Así como existen los actores sujeto, también existen los actores objetivo, los cuales deben ser vulnerables a los incentivos materiales o a las sanciones de los actores externos, o deben ser sensibles a la presión debido a las brechas entre los compromisos y la práctica declarados. Los países que son más susceptibles a las presiones de la red son aquellos que aspiran a pertenecer a una comunidad normativa de naciones (Keck \& Sikkink, 1998, pp. 28-29).

Retomando a Brysk (2007), hay ocasiones en las que el balance entre el Estado y el mundo puede cambiar gracias a acciones transnacionales, 
que a su vez afectan cómo actúa un Estado globalizado hacia sus propios ciudadanos. Brysk (2007) presenta una perspectiva en la cual la alianza entre las redes transnacionales de defensa y los pueblos indígenas representa un intento de equilibrar el campo de juego entre estos pueblos con sus gobiernos.

Khagram, Riker, y Sikkink (2002) sostienen que las instituciones internacionales presentan una clara estructura de oportunidad política para la incidencia transnacional. Nolan (2015) dice que las estructuras de oportunidad política son aquellos aspectos del entorno político interno, incluidas las instituciones, que promueven o limitan las acciones colectivas. Los movimientos sociales perciben estas estructuras como fuente de recursos para el apalancamiento político y espacios para el acceso a las estructuras políticas para influir en las políticas y el comportamiento del Estado. Las estructuras de oportunidades políticas transnacionales o internacionales se refieren a aquellos aspectos del entorno político internacional, incluidas las organizaciones internacionales, que pueden incentivar o restringir la acción colectiva. Las estructuras transnacionales no reemplazan a las nacionales, sino que se complementan e interactúan con estas, de manera que influyen en cómo los movimientos sociales desarrollan sus estrategias para incidir en el comportamiento del Estado a través de dos niveles de acción interconectados.

Martí y Silva (2014) mencionan los "cuatro tipos de interacción entre la estructura de oportunidades y amenazas internacionales y domésticas, así como diversos patrones de activismo" (Martí \& Silva, 2014, p. 13). Estos cuatro tipos son los siguientes:

1. Estructura de oportunidades internacional cerrada con una estructura doméstica cerrada, por ejemplo, se inhibe el activismo(Martí \& Silva, 2014, pp. 13-14).

2. Estructura doméstica cerrada, pero estructura de oportunidades política internacional abierta, por ejemplo, se generan formas clásicas de activismo, conocidas como patrón boomerang1 " Los activistas usan las redes transnacionales para presionar a los gobiernos nacionales que en un principio no son permeables a sus demandas" (Martí \& Silva, 2014, pp. 13-14).

1 La idea del boomerang proviene de que se lanza el objeto para arriba y luego se devolverá con alguna presa o premio; de la misma manera funcionaría el patrón de activismo transnacional. 
3. Estructura de oportunidades doméstica abierta con una estructura de oportunidades internacional cerrada: se genera un déficit democrático en el sistema internacional que incentiva un activismo transnacional defensivo (Martí \& Silva, 2014, pp. 13-14).

4. Estructura de oportunidades abierta en el ámbito doméstico y en el internacional: se promueven coaliciones que combinan el uso de protestas por parte de militantes con el activismo transnacional en los canales de política institucionalizada (Martí \& Silva, 2014, pp. 13-14).

Teniendo en cuenta lo anterior, en los estados federales - como es el caso de México- sería importante evaluar dichas estructuras de oportunidades políticas con base en el ámbito doméstico en lo nacional y local, dado que las entidades federales constituyen un punto clave de actividad política. En el presente estudio, buscamos analizar las interacciones entre actores sociales, gobiernos y actores internacionales no solamente a partir del impacto federal, sino en lo local para poder reflejar de forma más precisa la realidad política del país. De esta forma, buscamos aportar a la discusión teórica sobre las interacciones entre la esfera doméstica y la esfera internacional en el sentido de que se abra la esfera doméstica a la importante cuestión de la distribución territorial del poder. Esta perspectiva se vuelve aún más relevante cuando se contempla la distribución de los afrodescendientes en ciertos estados de la República, como se verá a continuación.

\section{Contextualización}

Por primera vez, el Instituto Nacional de Estadística y Geografía (Inegi) incluyó dentro de la Encuesta Intercensal del 2015 una pregunta con enfoque de autorreconocimiento a la población mexicana para identificar a la población afrodescendiente (Inegi, 2017). Parte de los resultados que arrojó esta pregunta fue que, de la población nacional, las personas que se consideran afrodescendientes suman 1,4 millones y representan el $1.2 \%$ de la población total (Inegi, 2015, p. 77). En la encuesta también se obtuvieron datos socioeconómicos sobre la población afromexicana de los cuales antes no se tenía registro ${ }^{2}$.

2 La transcendencia de la inclusión de esta pregunta en la Encuesta Intercensal se analizará más adelante. 
Durante el periodo colonial arribaron a México miles de personas traídas de manera forzada como esclavos, principalmente de regiones como Guinea, Mozambique, Congo y Angola (Velázquez \& Iturbide, 2012, p. 62). Los esclavos llegaban al país principalmente por los puertos de Veracruz, Campeche y Acapulco (Velázquez, 2016, p. 79). Por lo mismo, aún hoy en día la mayoría de la población afrodescendiente se ubica principalmente en Veracruz, así como en la Costa Chica de Guerrero y Oaxaca, ubicada en la llanura costera del Pacífico, desde el sur de Acapulco, Guerrero, hasta Huatulco, Oaxaca.

Aunque el movimiento afromexicano es relativamente reciente, la organización afrodescendiente en México se puede observar desde la época colonial. Ejemplo de esta organización son los cimarrones. El abuso físico y psicológico ocasionaba que los esclavos huyeran de las haciendas o plantaciones como forma de resistencia. A los esclavos que huían se les conocía como cimarrones y se asentaban en zonas aisladas denominadas palenques (Velázquez \& Iturralde, 2012, p. 73).

Por otro lado, en Coahuila se encuentra un grupo de afromexicanos definido como mascogos. A diferencia de todos aquellos que llegaron durante la colonia, los mascogos arribaron al país a mediados del siglo xix. Este pueblo resulta del intercambio y convivencia entre las poblaciones de seminoles (provenientes principalmente de Texas) y de los cimarrones (CDI, 2012, p. 42). Como recompensa por sus servicios de defensa durante la guerra de independencia, a finales de 1851 se les autorizó territorio en el interior del estado de Coahuila donde se les asignaron cuatro sitios de ganado en El Nacimiento, lugar donde habitan hasta hoy en día (CDI, 2012, p. 42).

Aunque en el estado de México, la Ciudad de México, Baja California Sur y Nuevo León hay gran cantidad de población afrodescendiente, estos no son considerados asentamientos históricos, pues son más bien factores demográficos actuales, como el intercambio migratorio, los que han ocasionado que el porcentaje de esta población sea alto (Inegi, 2017, p. 4).

Para Tarrow (1997), los movimientos sociales se conforman de cuatro propiedades principales, las cuales en el caso de los afromexicanos son las siguientes: tener objetivos comunes (cada asociación tiene diversos objetivos), tener un mismo desafío colectivo (el cual consideran que es su reconocimiento en la Constitución Federal de México), ser solidarios entre ellos (a pesar de sus diferencias tratan de colaborar en la creación de foros, congresos, paneles, etc.) y mantener una interacción constante entre ellos y las autoridades - los afromexicanos buscan apoyo constante 
de organismos como el Consejo Nacional para Prevenir la discriminación (Conapred), la Comisión Nacional de Derechos Humanos (CNDH), y la Comisión Nacional para el Desarrollo de los Pueblos Indígenas (CDI) - . A pesar de contar con tres de las propiedades, el movimiento afromexicano, como tal, aún no está fuertemente consolidado, pues las asociaciones carecen de objetivos comunes, convirtiéndolos en más que un movimiento en activistas en pro del reconocimiento de los afromexicanos.

En lo regional, a finales del siglo xx diversos países de América Latina tuvieron cambios en sus constituciones respecto al reconocimiento de los derechos de los afrodescendientes, en los cuales influenciaron las redes de activismo transnacionales. Como ejemplo tenemos el caso de Colombia. La Constitución Política de Colombia promulgada en 1991 significó un gran avance en términos jurídicos para la población afrodescendiente del mismo país. La constitución se sustentó en un paradigma multicultural y dentro de esta "la población 'negra' empieza a gozar de un marco legal que se refuerza con la Ley 70 de 1993 que les reconoce como un otro constitutivo de la personalidad de la Nación” (Valencia, 2015, p. 22). El Proceso de Comunidades Negras (PCN) es un actor-red que cuenta con alrededor de 120 organizaciones de base y de ley en los ámbitos regional y nacional (Espinosa-Bonilla, 2011, p. 218). Los procesos promulgados por el PCN hacen que se conecte con diversidad de redes transfronterizas (Europa, América Latina y Estados Unidos), pero también se transforme en un actor-red con una capacidad extraordinaria de relacionamiento e incidencia (Espinosa-Bonilla, 2011).

Por otro lado, de acuerdo con García y Benítez (2013), los nuevos movimientos sociales en México se pueden entender desde dos procesos complementarios: en primer lugar, el contexto global, relacionado con la transformación estatal de las últimas décadas del siglo $\mathrm{xx}$, y, en segundo lugar, un proceso que es particular del caso mexicano. En este contexto, cabe destacar que a partir de la década 1990, comunidades de la Costa Chica de Guerrero y Oaxaca se comenzaron a organizar y surgieron asociaciones sociales que demandan el reconocimiento de la presencia y contribuciones de la población afrodescendiente, así como "la vigencia de sus derechos para mejorar sus condiciones de vida” (Velázquez \& Iturbide, 2012, p. 21).

Este surgimiento se dio gracias a diversos factores. Por un lado, en la década de 1990 — en sintonía con diversos estados latinoamericanos - se dio una serie de políticas que surgieron en torno a la "relevancia de las reivindicaciones étnicas y de un salto en las dinámicas de reconocimiento institucional de la diversidad cultural en la región" 
(Agudelo, 2010, p. 112). Por otro lado, las características históricas de la Costa Chica ayudaron a que apareciera el movimiento de pueblos negros. Aún más importante, el factor que más resaltan algunos autores es que en diversos países de Latinoamérica, las políticas que los movimientos afrodescendientes comenzaron a tener y las demandas que hacían cobraron cierta importancia, que aumentó en México gracias a líderes sociales, así como académicos de la región. Este conocimiento arribó a México de diversas formas, a través de coloquios, encuentros, y congresos, entre otras actividades.

El movimiento afromexicano emergente fue principalmente impulsado por el padre Glyn Jemott Nelson. Originario de Trinidad y Tobago, él se encargó de realizar el Primer Encuentro de Pueblos Negros en 1997. Gracias a este evento, surgió México Negro, A. C., organización pionera en impulsar el movimiento afromexicano y actualmente una de las más fuertes (Castro \& Peñaloza, 2016, p. 123). Hoy en día, existen aproximadamente 10 organizaciones civiles dedicadas a la promoción y defensa de los derechos de las personas afromexicanas, las cuales tiene distintos enfoques y objetivos.

Hoffmann y Lara (2012) sostienen que la movilización local comenzó de diversas formas, algunos interesados en la recuperación y promoción de una identidad local; otros se enfocaban en la expresión de prácticas culturales como poesía, programas de radio o festivales de danza y música. Aunque este tipo de iniciativas se desarrollaron con motivos localizados diferentes, y no precisamente raciales o étnicos, hoy en día se proclaman como portavoces de la población negra o afro (Hoffmann \& Lara, 2012, p. 30).

A través de una entrevista realizada el 4 de noviembre del 2017 a la activista afromexicana Medhin Tewolde Serrano parece que un reto presente actualmente en el movimiento afromexicano para consolidarse como tal, es el autorreconocimiento de la misma población. Medhin es una mujer que se autoidentifica como afromexicana o más bien "afrodiaspórica”, al ser hija de padre proveniente de Eritrea y madre mexicana. Ella también mencionó: “a diferencia que en otros países de América Latina, en México la identidad afro creo que no está construida desde las bases y que se está construyendo más desde un discurso, el diálogo con el estado” (M. Tewolde, entrevista, 4 de noviembre, 2017).

A pesar de las diferencias entre los activistas afromexicanos y la naturaleza emergente del movimiento, se han producido varios logros de cara a su reconocimiento legal y visibilidad política. Dentro de su 
esfuerzo por consolidarse como un movimiento social, diversos activistas afromexicanos y organizaciones de la sociedad civil han recurrido a actores e instrumentos internacionales que pueden brindarles apoyo para lograr sus objetivos en lo nacional y local. A continuación se estudió el activismo transnacional del movimiento afromexicano tanto en la esfera federal como en la esfera estatal, con base en el análisis de entrevistas en profundidad, notas de prensa, normas legales, informes oficiales y otra documentación.

\section{Análisis de caso}

Es importante recordar que desde que comienza la formación del movimiento afromexicano en la década de 1990, se dio un primer contacto con actores internacionales para la creación de una red transnacional, a través del Padre Glynn Jemmott Nelson, quien llegó a la región de Costa Chica en 1984 (Miranda, 2012, p. 84). Sin embargo, fue hasta mediados de la siguiente década que el activismo afromexicano comenzó a crecer y se impulsó al pueblo afromexicano a reconocerse como tal. Así, una vez establecidos vínculos con el sector académico, los afromexicanos comenzaron a tejer redes transnacionales enfocadas a la defensa de los derechos y el reconocimiento de estos dentro de la constitución federal de México (Peñaloza-Pérez, 2017). En este caso se analizará varios procesos en los que se ha activado una red de activismo transnacional por parte del movimiento afromexicano y su impacto federal y local.

\section{Impacto federal}

Aunque el poder legislativo se ha mostrado renuente a reconocer a los afromexicanos como pueblo dentro de la Constitución Política de los Estados Unidos de México, se ha producido un debate muy interesante al respecto y además se han producido varios logros en otras esferas nacionales. A continuación, se relata y evalúa con detalle la lucha del movimiento afromexicano por el reconocimiento político federal, haciendo hincapié en la influencia de aliados y normas internacionales (incluyendo la Conferencia de Durban y el Decenio Internacional de los Afrodescendientes) sobre el Gobierno mexicano. En primer lugar, se analizará la presión para lograr el reconocimiento constitucional para después seguir con otras esferas de reconocimiento de corte más simbólico, en ambos casos a contexto federal. 


\section{Presión para lograr el reconocimiento en la Constitución Política de los Estados Unidos de México}

Como primera demanda y más relevante, se encuentra la reivindicación del pueblo afromexicano en la carta magna de México. Aunque las organizaciones de activistas afromexicanos están dispersas como base, algo que todos demandan (aunque desde distinto enfoque) es el reconocimiento constitucional. Líderes afromexicanos como Rosa María Castro-Salinas y Sergio Peñaloza-Pérez (2016) argumentan que la población afromexicana considera el Decenio Internacional de los Afrodescendientes (2015-2024) como el marco idóneo para exigir el cumplimiento de los postulados que proclama. Con el inicio del Decenio en el 2015, las demandas por el reconocimiento constitucional de los afromexicanos se fueron haciendo más visibles. Por ejemplo, en el 2015 la población de Mascogos en Múzquiz, Coahuila denunció ante la $\mathrm{CNDH}^{3}$ la falta del reconocimiento constitucional ("Afromexicanos denuncian en CNDH falta de reconocimiento constitucional”, 2015). Simultáneamente, en el sur de México, específicamente Oaxaca, el Consejo Consultivo de los Pueblos Indígenas y Afromexicanos de Oaxaca lanzó un manifiesto a través del Centro de Derechos Humanos Miguel Agustín Pro Juárez A. C. ${ }^{4}$. En el marco de este manifiesto, se hacen demandas al Gobierno federal para que tome en cuenta todos sus compromisos internacionales para el reconocimiento de los pueblos aquí mencionados. Al final, hacen un llamado a la comunidad internacional, estableciendo cada vez más presión hacia el Estado mexicano.

Un momento clave en este proceso fue la participación de un grupo de afrodescendientes mexicanas en la Primera Cumbre de Lideresas Afrodescendientes de las Américas realizada en Nicaragua, Managua en el 2015. En ocasiones las redes transnacionales vinculan activistas de países desarrollados con otros que están en desarrollo o que simplemente son menos desarrollados (Keck \& Sikkink, 1999). Así, las activistas afromexicanas se dieron cuenta que a través de esta Cumbre, que reúne a lideresas de países con más avances en la temática afrodescendiente, pueden llegar a tener algún impacto en las acciones del Gobierno mexicano. En tal evento, la licenciada Rosa María Castro-Salinas señaló

\footnotetext{
3 Comisión Nacional de Derechos Humanos

4 Organización no gubernamental con estatus consultivo roster en el Consejo Económico y

Social de las Naciones Unidas y acreditada ante la Organización de Estados Americanos.
} 
que, a pesar de que no todos los países de América Latina reconocen en su carta magna a la población afrodescendiente, sí se reportan más avances de recuperación histórica y de identidad que en México (Soto, 2015).

En este evento se realizó una misiva firmada por la coordinadora general de la cumbre, así como las coordinadora de zonas como el Cono Sur, la Diáspora, la región Andina, el Caribe y la líder para Centroamérica. En la misiva, dirigida a Enrique Peña Nieto, con copia al Congreso de la Unión, se le exigía "las acciones necesarias para lograr la reivindicación de los derechos fundamentales de la población afromexicana" (Soto, 2015). Además, se pidió reformar el artículo 2 de la Constitución, para establecer que "[...] la Nación tiene una composición pluricultural sustentada principalmente en sus pueblos indígenas y afrodescendientes" (Soto, 2015). De la misma forma, se le envió una carta al presidente con los acuerdos de la Cumbre en la que se exhorta al Gobierno mexicano a que "[...] otorgue al Pueblo Negro de México el reconocimiento constitucional inmediato" ("Piden implementar políticas públicas a favor de afromexicanos", 2015a). Esta carta representa un punto clave que comienza a crear mayor presión hacia el Estado.

En el mismo año, en el marco del foro "Estudios Internacionales, México 2015. Una mirada hacia África: Presente, Integración y Futuro“, el Senado y la Secretaría de Gobernación (Segob) impulsaron una reforma constitucional para reconocer los derechos de las personas que conforman los pueblos afromexicanos o afrodescendientes en el país ("Senado y Segob promueven reconocimiento constitucional de pueblos afromexicanos", 2015). Se subrayó que el mayor problema que este pueblo enfrenta en el país es que no están reconocidos en la Constitución, haciendo que no tengan derechos a la libre determinación y que enfrenten dificultades para acceder a los programas sociales. Luis Sánchez Jiménez, vicepresidente de la Mesa Directiva del Senado, llamó a que se reconociera y se hiciera visible ante la sociedad mexicana a la raíz africana, teniendo en cuenta sus necesidades y actuando siempre a favor del reconocimiento de los derechos humanos ("Senado y Segob promueven reconocimiento constitucional de pueblos afromexicanos", 2015).

Más adelante, cuando se presentó el Informe de organizaciones de población afrodescendiente de América Latina 2016 de la Secretaría General Iberoamericana, el Programa de Naciones Unidas Para el Desarrollo y la Agencia Española de Cooperación Internacional para el Desarrollo se pronunciaron en favor del reconocimiento de los derechos individuales y políticos de la población afromexicana ("Reconocer los 
derechos de la población afromexicana, urge el Conapred”, 2017). El Conapred ofreció acompañamiento a la población afromexicana con los legisladores, promoviendo el reconocimiento constitucional de la población afrodescendiente. Además del Conapred, estuvieron presentes representantes de la CNDH, académicos del Instituto Nacional de Antropología e Historia (Inah), el Senado de la República, y la Fundación Afromexicana Petra Morga, A.C ("Reconocer los derechos de la población afromexicana, urge el Conapred”, 2017).

En los últimos años se ha producido una serie de iniciativas para reformar la Constitución Política de los Estados Unidos de México de cara al reconocimiento de la población afrodescendiente (Velásquez \& Iturralde, 2016). La última fue a principios del 2017, cuando la diputada Laura Beatriz Esquivel Valdés, del partido Morena, presentó una iniciativa que reforma los artículos 2. $, 27,28$ y 155 de esta. Esta iniciativa insta al Estado a retomar los diversos convenios y recomendaciones internacionales que México ha firmado sobre el combate al racismo, entre las cuales destaca la declaración del 2011 como el año internacional de los afrodescendientes, así como la declaración del Decenio Internacional de los Afrodescendientes (2015-2024). A pesar de que existe el reconocimiento de los afromexicanos en distintos contextos, el reconocimiento constitucional de estos pueblos es importante, pues la Constitución Política de los Estados Unidos de México establece que México es un país pluricultural, "[...] lo que implica que todas las culturas que están dentro de la nación, deben tener un reconocimiento al mismo nivel” (Esquivel, 2017). Por lo tanto, reformar los artículos antes mencionados aportan a "[...] reconocer e incluir en el texto constitucional los derechos de los pueblos negros afromexicanos" (Esquivel, 2017).

Es importante destacar que, a pesar de la evidente presión ejercida por parte de actores locales, internacionales, gubernamentales y políticos en el marco del Decenio Internacional de los Afrodescendientes, el reconocimiento de los afromexicanos en la Constitución Política de los Estados Unidos de México aún no se ha producido. Se considera que es así por dos motivos principales: en la nación, el movimiento afromexicano todavía está en proceso de consolidación, e internacionalmente, la falta de presión por parte de actores más influyentes, como otros estados, retrasa el proceso. Aunque no se aprecia presión clara por parte de otros estados, existe presión por parte de la sociedad civil (p. e. la carta realizada al Presidente Enrique Peña Nieto en la Primera Cumbre de Lideresas de las Américas), presión por parte de actores de Gobierno y nacionales, 
entre otros, lo que hace que no se descarte la posibilidad de que en un futuro cercano se les otorgue a los afromexicanos su reconocimiento constitucional en el Contexto federal. En ese sentido, es importante recordar que, para que se produjera el reconocimiento constitucional federal de los pueblos indígenas en México, pasaron más de siete años entre el levantamiento zapatista (1994) y la reforma al artículo 2 de la Constitución Política (2001), y que existe cierta resistencia a la hora de reconocer derechos colectivos, sobre todo cuando tienen implicaciones políticas (Wright, 2018). Entonces, la variable del timing es sumamente importante, y, en caso de los afromexicanos, podremos afirmar que la masa crítica de presión tanto desde adentro como desde afuera de México no ha llegado a niveles suficientes como para propiciar un cambio.

Se debe tener en cuenta que el movimiento afromexicano apenas comienza un proceso de autoidentificación y generación de una identidad propia; esto hace que, a pesar de que académicos hablen del tema y se luche por el reconocimiento de los afromexicanos, al estar ellos dispersos, no logran ser una base sólida que pueda presionar lo suficiente para lograr este reconocimiento. Dentro de la antes mencionada entrevista a Medhin Tewolde, ella considera que, aunque es de suma importancia que los pueblos negros de México sean reconocidos constitucionalmente, el proceso no se está haciendo desde las bases, sino que hay un discurso afro en México que se hace desde arriba y luego baja a las comunidades, y esto tendría que ser al revés (Tewolde, entrevista, 4 de noviembre, 2017).

\section{Una creciente visibilidad institucional}

Si bien aún no se ha dado el reconocimiento constitucional, que por su naturaleza tomaría, en caso de darse, cierto tiempo, algunas instancias gubernamentales han elaborado planes de trabajo en el marco de la Conferencia de Durban y del Decenio Internacional de los Afrodescendientes (2015-2024).

En el 2015, México firmó el acuerdo del Decenio, en el cual se insta a los estados a que atiendan a las resoluciones de la Conferencia de Durban para realizar medidas concretas como "[...] recoger, recopilar, analizar, difundir y publicar datos estadísticos para evaluar periódicamente la situación de los afrodescendientes" (Inegi, 2017, VIII):

De acuerdo con estas orientaciones, atendiendo la demanda de diversas organizaciones sociales de afrodescendientes, de instituciones de los tres órdenes de Gobierno, de legisladores y de investigadores 
interesados en el tema, el Inegi incluyó una pregunta en la Encuesta Intercensal 2015 [...] para identificar a la población afrodescendiente en México, bajo el enfoque de autorreconocimiento (Inegi, 2017, VIII).

La pregunta textual que se encontraba dentro de la Encuesta Intercensal 2015 fue "[d]e acuerdo con su cultura, historia y tradiciones, $¿[\ldots]$ se considera negra(o), es decir, afromexicana(o) o afrodescendiente?" (Inegi, 2015). Entre los principales resultados se encuentra que las personas que se autorreconocen como afromexicanos suman 1,4 millones de personas, lo que representa el 1,2\% de la población nacional (Inegi, 2015, p. 77). Por lo tanto, sin duda alguna el reconocimiento de la población afrodescendiente en la Encuesta Intercensal 2015 es un paso absolutamente clave en la política de reconocimiento del pueblo afromexicano.

Por su parte, instituciones como el Conapred, la CDI y la CNDH han sido los principales receptores de la presión por parte de actores internacionales al ser instituciones relacionadas con la defensa de los derechos humanos y de las minorías. Así, han generado iniciativas y literatura respecto a los afromexicanos, la cual va haciendo más notoria la necesidad del reconocimiento de la población afromexicana y presiona al gobierno mexicano para que tome acciones concretas. A su vez, esta visibilidad institucional es prueba de que el Estado le está poniendo atención a las normas internacionales y, por lo tanto, tiene cierto grado de susceptibilidad frente a redes de activismo tanto internacionales como nacionales.

Uno de los principales actores clave de cara a la visibilidad de la población afromexicana ha sido el Conapred. Se creó en el 2003 para intentar responder a los acuerdos firmados en Durban por parte del Gobierno mexicano. El Conapred es una "[...] instancia del Estado que ha sido uno de los principales canales de interlocución para dar seguimiento a las demandas de las organizaciones negras" (Hoffmann \& Lara, 2012, p. 15). A lo largo de los años se ha enfocado a crear recomendaciones e iniciativas con el fin de fortalecer su identidad, y promover la interculturalidad así como proponer acciones para erradicar y combatir la discriminación (Conapred, 2010).

Eva Victoria Gasga (2013) considera que lo afrodescendiente se convirtió en un tema de la agenda nacional a partir del 2011 en el marco del año internacional de los afrodescendientes. Así, retomando los compromisos hechos por México dentro de un marco de 
referencia internacional, la CDI realizó en el 2012 una consulta para la identificación de las comunidades afrodescendientes de México: Coahuila, Guerrero, Oaxaca, Veracruz, Chiapas y Michoacán; esto a partir de las particularidades de cada estado. La consulta sirvió como antecedente a la pregunta incluida en la Encuesta Intercensal 2015 así como para identificar y generar información útil para conocer más sobre la población afrodescendiente (CDI, 2012, p. 21).

Por su parte, en junio del 2015, la CNDH presentó el plan de trabajo de México en torno al Decenio. Este incluía acciones legislativas, así como relacionadas con la igualdad y la no discriminación, la educación, la producción y difusión de información estadística y de investigación, entre otras acciones encaminadas a impulsar la inclusión de la comunidad afromexicana (CNDH, 2016, pp. 15-16).

En adición a los esfuerzos institucionales, y reconociendo una larga lista de foros y eventos organizados desde la década de los 1990 por el movimiento afromexicano, es importante referirnos también a dos eventos o procesos celebrados con actores internacionales que han servido para fortalecer la visibilidad afrodescendiente a Contexto federal en México. Un evento de gran transcendencia para la visibilidad de la población afrodescendiente en México fue la organización del Segundo Coloquio Internacional de Afrodescendientes, del cual fue sede México y se realizó a finales del 2015 (Copred, 2015). Su objetivo fue poner en práctica estrategias internacionales, regionales y nacionales que promuevan "[...] la inclusión de las personas afrodescendientes y superar el racismo y la discriminación racial, la xenofobia y las formas de intolerancia en el mundo" (Copred, 2015).

En mayo del 2017, dentro del contexto del Decenio, el Inah, en conjunto con el Centro de Investigaciones y Estudios Superiores en Antropología Social y la Organización de las Naciones Unidas para la Educación, la Ciencia y la Cultura (Unesco) abrieron la Cátedra Unesco Afrodescendientes en México y Centroamérica: Reconocimiento, Expresión y Diversidad Cultural. La cátedra se divide en tres ejes principales: “[...] la investigación histórica y contemporánea sobre poblaciones afrodescendientes, la formación de recursos humanos interesados en las problemáticas de este grupo" (Secretaría de Cultura, 2017), y crear un programa de divulgación que aporte a sensibilizar a los responsables de las políticas públicas sobre la importancia de fomentar una cultura de paz que acabe con el racismo (Secretaría de Cultura, 2017). 
Por todo lo anterior, queda claro que, a partir del Año Internacional de los Afrodescendientes en el 2011 y, especialmente, de cara al inicio del Decenio Internacional de los Afrodescendientes en el 2015, se ha producido una acelerada y amplia visibilidad de los afrodescendientes en México, debido en gran medida a la influencia conseguida por activistas afromexicanos con aliados y normas internacionales, autoridades gubernamentales sensibles a la temática, en un momento en el mundo que se puede identificar como altamente favorable. Si bien no equivale a un reconocimiento formal como sujeto de derechos, estos desarrollos recientes son prueba de que los afromexicanos se encuentran en la agenda pública y en un proceso amplio de reconocimiento sociopolítico a Contexto federal.

\section{Impacto estatal}

A pesar de que el reconocimiento constitucional en la carta magna de México se considera como la principal reivindicación del movimiento afromexicano, es importante destacar que ha habido avances considerables en las constituciones de aquellos estados con mayor población afrodescendiente. Los afromexicanos son reconocidos en las constituciones políticas de los estados de Oaxaca, Guerrero y la Ciudad de México. Si bien dicho reconocimiento se ha logrado principalmente gracias a esfuerzos de activistas locales e instituciones gubernamentales, la presión de organismos y ONG internacionales, así como la influencia ofrecida por el Decenio de los Afrodescendientes fueron claves para lograr el reconocimiento. En todos los casos es importante señalar que las reformas se dieron de la mano con cambios para profundizar los derechos políticos de los pueblos indígenas, situación que sugiere que, al tratarse de dos grupos de pueblos, se pudo llegar a la masa crítica como para poder abrir una ventana de oportunidades políticas favorables locales. A continuación, se detallara más del proceso que se dio en cada estado.

A pesar de que en 1990 se incluyó de forma discreta al pueblo afromexicano dentro del artículo 3. ${ }^{\circ}$ de la Constitución Política del Estado Libre y Soberano de Oaxaca, en la cual se les otorga el libre ejercicio periodístico en cualquier medio de comunicación, fue hasta 1998 que Oaxaca se reconoció como un estado de composición étnica plural debida a la diversidad de los pueblos y comunidades que lo integran, incluyendo las comunidades afromexicanas. Sin embargo, en el 2013 Oaxaca se 
convirtió en el primer estado en aprobar el artículo $10^{\circ}$ que prohíbe la discriminación, el racismo y la xenofobia (Castro, 2017). Más adelante, en el 2014, el gobernador Gabino Cué presentó al Congreso del Estado la Reforma sobre los derechos de los pueblos indigenas y el pueblo afromexicano de Oaxaca, "[...] resultado de un amplio proceso de consulta, diálogo, análisis y reflexión, a través de foros regionales y estatales, en el que han participado de manera activa los 15 pueblos indígenas y el pueblo negroafromexicano de Oaxaca" (Oxfam México, 2015a).

A lo largo de este proceso es importante destacar el papel de ONG internacionales como Oxfam. Concretamente, junto con diversas organizaciones indígenas y afrodescendientes — el Consejo Consultivo Indígena y Afromexicano de Oaxaca, el Centro de Derechos Indígenas Flor y Canto, la Asamblea Mixe para el Desarrollo Sostenible A. C., la Coordinadora de Pueblos Unidos por el Cuidado y Defensa del Agua, el Frente de Autoridades Municipales y Agrarias por el Cuidado y Defensa del Territorio del Valle de Oaxaca—, esta ong lanzó la campaña “¿Qué pasó con mis derechos?" para la aprobación de la Reforma sobre los derechos de los pueblos indígenas y el pueblo afromexicano de Oaxaca (Oxfam México, 2015b). Así, en el 2015 se aprobó finalmente la reforma constitucional en el estado de Oaxaca, para fortalecer "[...] a los pueblos originarios, así como las posibilidades de los pueblos indígenas y afromexicanos como entidades colectivas y sujetos de derecho para que escojan 'las opciones de vida que autónomamente valoren"” (E-Oaxaca, 2015).

En el caso de Guerrero, se dio una reforma integral a la constitución estatal, que incluía un claro reconocimiento del pueblo afrodescendiente. En el marco de la celebración del Día Internacional de los Pueblos Indígenas en el 2011, la Secretaría de Asuntos Indígenas realizó un foro de análisis sobre los pueblos indígenas y afromexicano en la constitución, para incluir sus derechos y cultura en la nueva constitución política del estado libre y soberano de Guerrero en Chilpancingo, Guerrero (Secretaría de Asuntos Indígenas, 2011). Además, ese año se llevó a cabo el foro estatal Capítulo Indígena y Afromexicano en la Nueva Constitución Política del Estado Libre y Soberano de Guerrero. De forma interesante, Gonzalo Ramón Solís Cervantes, consejero de la CDI que se autorreconoce como negro, observó que la idea de buscar el reconocimiento del pueblo afrodescendiente surgió en el 2011, cuando las Naciones Unidas celebró el año de los afrodescendientes, y "[...] de ahí surgió la idea de exigir el reconocimiento constitucional porque se nos escondía en el mestizaje, se nos llamaba afromestizos, decidimos nombrarnos afrodescendientes 
a nivel global, afromexicanos y afroguerrerenses" ("Reconocerá la nueva Constitución al pueblo afro como la quinta etnia de la entidad”, 2012). Esta propuesta fue impulsada por la Subsecretaría para el Desarrollo del Pueblo Afromexicano y se incorporó dentro de la reforma integral a la constitución local aplicada en el 2014.

Dentro del segundo capítulo de la Constitución Política del Estado Libre y Soberano de Guerrero (2014), denominado "Derechos Humanos y Garantías", se encuentra la sección "De los derechos de los pueblos indígenas y afromexicanos", la cual comprende del artículo $8 .^{\circ}$ al $14 .^{\circ}$, en los cuales el estado de Guerrero reconoce y garantiza los derechos de los pueblos indígenas y afromexicano. Los derechos que principalmente se les reconoce son aquellos relacionados con su libre determinación y autonomía, así como el acceso a la jurisdicción del Estado, teniendo en cuenta sus usos, costumbres y demás aspectos culturales. Nuevamente, es importante recalcar que los eventos mundiales que dieron impulso a todo este proceso fue tanto la celebración del Día Internacional de los Pueblos Indígenas en el 2011 como el año de los afrodescendiente, que abrió una ventana de oportunidad favorable para impulsar el reconocimiento de los afrodescendientes en el Estado.

El proceso para formular una Constitución para la Ciudad de México fue muy largo y debatido. Un punto importante del debate fue el de los derechos de las minorías sociales, incluyendo los indígenas, los migrantes y los afromexicanos. Sobre este último punto, que es el que nos concierne en este escrito, es importante destacar que fue promovido a través de un Decálogo para el reconocimiento de las poblaciones afromexicanas y sus derechos en la Constitución Política de la Ciudad de México, elaborado por organizaciones de la sociedad civil, que incluían Afrodescendencias en México Investigación e Incidencia A. C. y México Negro, entre otras ${ }^{5}$. De las diversas razones por incluir el reconocimiento de los afromexicanos en la nueva constitución, dentro de las cuales estaban su presencia histórica en la ciudad, así como la continua discriminación en su contra, se encuentran los compromisos internacionales asumidos por el Estado mexicano en el marco de la Conferencia de Durban y el Decenio de los Afrodescendientes (Masferrer \& Ramírez, 2016). Por su parte, la Comisión de Derechos Humanos del Distrito Federal dedicó el número 7, año XV,

5 La petición se puede revisar en la siguiente liga: https://www.change.org/p/diputadas-ydiputados-de-la-asamblea-constituyente-de-la-ciudad-de-m\%C3\%A9xico-reconocimiento-de-laspoblaciones-afromexicanas-en-la-constituci\%C3\%B3n-de-la-ciudad-de-m\%C3\%A9xico 
de Dfensor, su revista mensual precisamente al tema del reconocimiento de los derechos de las personas indígenas, en el que varios autores hacen hincapié en la oportunidad ofrecida por el Decenio.

Como resultado de todo lo anterior, dentro de la Constitución Política de la Ciudad de México, aprobada a principios del 2017, se incluye a las personas afrodescendientes en la fracción N del artículo 11, denominado "Ciudad incluyente". En este apartado se les garantiza a estos individuos el goce de los derechos reconocidos por la misma constitución. Además, “[...] reconoce y protege las contribuciones históricas de las personas afromexicanas en la construcción de la nación mexicana y de la Ciudad de México" (Constitución Política de la Ciudad de México, 2017, art. 38).

Los logros conseguidos en el reconocimiento de los afrodescendientes en el contexto estatal constituyen un punto de referencia para el movimiento afromexicano en su reivindicación constitucional federal. Tanto así que en Veracruz actualmente se están discutiendo un proyecto de reforma constitucional (García, 2017). A través de largos procesos de reivindicación e incidencia política por parte de organizaciones locales, con el apoyo de actores internacionales o la influencia ofrecida por eventos internacionales como el Año o el Decenio de los Afrodescendientes, se logró ejercer suficiente presión sobre los congresos locales como para producir transformaciones significativas en las constituciones locales. Sin duda alguna, parece que las autoridades locales han sido más permeables a esta presión que las autoridades federales. Otro factor para considerar es que en los tres casos el reconocimiento de derechos afrodescendientes se logró de forma conjunta con la población indígena, situación que no ocurrió en el contexto federal. De esta forma, se puede hablar de una masa crítica de presión en lo local que ha influido de forma decisiva para lograr cambios sustantivos.

\section{Conclusiones}

El objetivo del presente estudio ha sido analizar las interacciones del movimiento afromexicano con una red de defensa transnacional, sus derechos, así como los impactos que han tenido dichas interacciones tanto en lo nacional como en lo local. Para guiar el análisis, se partió de la propuesta de estructuras de oportunidades políticas articulada por Martí y Silva (2014), si bien, debido a la estructura federal del sistema político 
mexicano, en este artículo se entiende el espacio doméstico como dividido entre lo federal y lo estatal.

Tabla 1. Logros del movimiento afromexicano en los distintos ámbitos del contexto nacional.

\begin{tabular}{|c|c|c|}
\hline & Logros & Actores o eventos que influyeron \\
\hline $\begin{array}{l}\text { Âmbito } \\
\text { federal }\end{array}$ & $\begin{array}{l}\text { Si bien no se ha logrado el reconocimiento } \\
\text { constitucional en el contexto federal, } \\
\text { existe una presión para lograr el } \\
\text { reconocimiento de los afromexicanos, que } \\
\text { cada vez va creciendo más, además de una } \\
\text { creciente visibilidad institucional. } \\
\text { Se toma en cuenta a la población } \\
\text { afromexicana dentro de la encuesta } \\
\text { Intercensal } 2015 \text { del Inegi. } \\
\text { La cDi realizó en el } 2012 \text { la consulta para } \\
\text { la identificación de las comunidades } \\
\text { afrodescendientes de México. }\end{array}$ & $\begin{array}{l}\text { Conapred, CNDH, INAH, Unesco, } \\
\text { influencia por parte del Decenio } \\
\text { Internacional de los Afrodescendientes, } \\
\text { así como la Conferencia de Durban. }\end{array}$ \\
\hline $\begin{array}{l}\text { Ámbito } \\
\text { estatal } \\
\text { (Oaxaca) }\end{array}$ & $\begin{array}{c}\text { En el } 2015 \text { se aprobó la reforma } \\
\text { constitucional en el estado de Oaxaca, } \\
\text { para fortalecer a los pueblos originarios, } \\
\text { así como las posibilidades de los pueblos } \\
\text { indígenas y afromexicanos. }\end{array}$ & $\begin{array}{l}\text { Oxfam, el Consejo Consultivo Indígena } \\
\text { y Afromexicano de Oaxaca, el Centro } \\
\text { de Derechos Indígenas Flor y Canto, } \\
\text { la Asamblea Mixe para el Desarrollo } \\
\text { Sostenible A. C., la Coordinadora de } \\
\text { Pueblos Unidos por el Cuidado y Defensa } \\
\text { del Agua, el Frente de Autoridades } \\
\text { Municipales, y Agrarias por el Cuidado y } \\
\text { Defensa del Territorio del Valle de Oaxaca }\end{array}$ \\
\hline $\begin{array}{c}\text { Ámbito } \\
\text { estatal } \\
\text { (Guerrero) }\end{array}$ & $\begin{array}{l}\text { En el } 2014 \text { se incorporó dentro del } \\
\text { segundo capítulo de la Constitución } \\
\text { Política del Estado Libre y Soberano de } \\
\text { Guerrero, la sección “De los derechos de } \\
\text { los pueblos indígenas y afromexicanos”. }\end{array}$ & $\begin{array}{c}\text { Se logró gracias a la influencia y dentro } \\
\text { tanto del marco del Día Internacional de } \\
\text { los Pueblos Indígenas en el } 2011 \text { como el } \\
\text { Año de los Afrodescendiente. }\end{array}$ \\
\hline $\begin{array}{l}\text { Ámbito } \\
\text { estatal } \\
\text { (Ciudad de } \\
\text { México) }\end{array}$ & $\begin{array}{l}\text { Dentro de la Constitución Política de la } \\
\text { Ciudad de México, aprobada a principios } \\
\text { del 2017, se incluye a las personas } \\
\text { afrodescendientes en la fracción N del } \\
\text { artículo 11, "Ciudad incluyente" }\end{array}$ & $\begin{array}{l}\text { ONG nacionales, como México Negro y } \\
\text { la influencia de eventos internacionales } \\
\text { como la Conferencia de Durban y el } \\
\text { Decenio de los Afrodescendientes. }\end{array}$ \\
\hline
\end{tabular}

Fuente: elaboración propia.

En primer lugar, se ha constado que existe una estructura internacional de oportunidades políticas abierta, después de años y décadas de movilización internacional sobre la discriminación y reconocimiento de la identidad étnica (incluyendo la afrodescendiente). Tal estructura abierta se ha plasmado en acuerdos y eventos internacionales, sobre todo a inicios del Decenio Internacional de los Afrodescendientes. Como señala Masferrer, “[e]l Decenio Internacional 
para los Afro descendientes (2015-2024) representa una oportunidad para saldar la deuda histórica que tenemos con la población afromexicana[...]" (2017, p. 15). También es importante destacar el papel de la Conferencia de Durban (Quintana \& Analís, 2017) y el Año Internacional de los Afrodescendientes del 2011 (Quintana \& Analís, 2017). De la misma forma, ha estado presente una gran gama de actores internacionales, desde órganos de las Naciones Unidas, Agencias Multilaterales de Desarrollo, Agencias Internacionales de Cooperación Internacional y ONG Internacionales. Si bien es emergente, el movimiento afromexicano ha articulado redes transnacionales en este contexto favorable, buscando ejercer presión sobre diversos actores poderosos, tanto en el contexto federal como en el local.

El reconocimiento constitucional ha sido la principal reivindicación del movimiento afromexicano, y, a pesar de una presión sostenida por parte de organizaciones afromexicanas, autoridades gubernamentales federales — sobre todo el Conapred y la CNDH—, del sector académico y aliados internacionales, incluso usando el Decenio Internacional de los Afrodescendientes como influencia, hasta la fecha no se ha conseguido un cambio en la Constitución Política de los Estados Unidos de México al respecto. Por otra parte, es importante reconocer que, en los últimos años, sobre todo a inicios del Decenio, se ha producido una creciente visibilidad de los afromexicanos en la esfera político-social federal, incluyendo una pregunta en el Censo del Inegi y varios eventos, estudios y cátedras organizados por las propias organizaciones afromexicanas, así como por instituciones gubernamentales, y en algunos casos con el apoyo de aliados internacionales. En el contexto local, la influencia conseguida por parte de organizaciones afromexicanas con autoridades gubernamentales y aliados internacionales ha sido lo suficientemente grande como para dar lugar al reconocimiento constitucional en tres estados de la República: Oaxaca, Guerrero y la Ciudad de México.

Por todo lo anterior, siguiendo el esquema de Martí y Silva (2014), podemos afirmar que, mientras que la estructura de oportunidades políticas se encuentra abierta en el ámbito internacional, existen diferencias en las interacciones con el movimiento afromexicano en el contexto federal y estatal. Por un lado, en el contexto federal empieza a pasarse de una estructura cerrada a una abierta, debido a una estrategia exitosa de vinculación entre organizaciones indígenas, actores internacionales y autoridades gubernamentales a cargo de la promoción de los derechos humanos y la no discriminación. Además, la reforma 
constitucional del 2011, que confirma que los tratados internacionales son vinculantes, es un paso más hacia dicha apertura. Por otro lado, en lo estatal la estructura de oportunidades más bien se encuentra abierta, debido a una larga historia de incidencia y articulación de demandas en el contexto local, y los eventos y aliados internacionales se han instrumentalizado de forma favorable para incidir en el reconocimiento constitucional. La gran diferencia entre ambas esferas domésticas tendría que ver con la configuración de la población indígena (que se concentra en ciertos estados), la influencia de las organizaciones afromexicanas en el contexto local y la permeabilidad de las instituciones gubernamentales a la presión de redes transnacionales de defensa. Otro hallazgo inductivo es que, en el contexto local, los cambios se han producido en el marco de reformas más amplias en las que estaba contemplada también una profundización de los derechos de los pueblos indígenas, un aliado estratégico clave.

Si bien el enfoque del presente estudio ha sido sobre la articulación de redes transnacionales y la influencia de normas internacionales en los contextos federal y estatal en México, no debe subestimarse el proceso histórico de articulación y presión por parte del movimiento afromexicano en sí, que sin duda es dueño de todos los logros conseguidos hasta la fecha, así como aquellos logros que en un futuro próximo podrían darse. La apertura de una estructura de oportunidades políticas más favorable en el contexto local sirve como fuerte antecedente e incluso mecanismo de presión para que la estructura a Contexto federal se torne, a su vez, más favorable.

\section{Referencias}

Afromexicanos denuncian en CNDH falta de reconocimiento constitucional. (2015). La Jornada Baja California. Recuperado de http://jornadabc. $\mathrm{mx} /$ tijuana/22-12-2015/afromexicanos-denuncian-en-cndh-falta-dereconocimiento-constitucional

Agudelo, C. (2010). Movilizaciones afrodesc'endientes en América Latina. Una visión panorámica de algunas experiencias contra la exclusión y por el derecho a la identidad. Recuperado de http://www.scielo.org.co/pdf/rci/n71/n71a06.pdf

Anaya, A. (2014). Los derechos humanos en y desde las Relaciones Internacionales. México: Centro de Investigación y Docencia Económicas.

Bello, A. (2004). Etnicidad y ciudadanía en América Latina: La acción colectiva de los pueblos indígenas. Santiago de Chile: Comisión Económica para América Latina y el Caribe. 
Brysk, A. (2007). Globalización y pueblos indígenas: el rol de la sociedad civil internacional en el siglo xxi. En S. Martí i Puig (ed.), Pueblos indígenas y política en América Latina (pp. 17-30). Barcelona: Fundación сгров.

Capítulo Indígena y Afromexicano en la Nueva Constitución. (2011). Gobierno del Estado Libre y Soberano de Guerrero. Recuperado de http://administracion2014-2015. guerrero.gob.mx/articulos/capitulo-indigena-y-afromexicano-en-la-nuevaconstitucion/

Castro, S. (2017). Acto Inaugural. Congreso Internacional Estudios Afromexicanos: reflexiones, debates y retos. 5 de septiembre, Oaxaca, México.

Castro-Salinas, R., \& Peñaloza-Pérez, S. (2016). El movimiento de pueblos negros de México a veinte años de su inicio: análisis y reflexiones. En H. Peña (ed.), Afromexicanos: Pertenencia y Orgullo (pp. 123-150). Ciudad de México: Comisión Nacional de los Derechos Humanos.

Comisión Economica para América Latina y el Caribe [Cepal]. (2000). Etnicidad, "Raza”y Equidad en América Latina y El Caribe. Recuperado de https://repositorio.cepal.org/bitstream/handle/11362/31450/S008674_ es.pdf?sequence $=2$

Comisión Nacional para el Desarrollo de los Pueblos Indígenas [CDI]. (2012). Informe final de la Consulta para la identificación de comunidades afrodescendientes. Ciudad de México: Comisión para el Desarrollo de los Pueblos Indígenas. Comisión Nacional de Derechos Humanos [CNDH]. (2016). Capacitan CNDH y Uabjo a mujeres afromexicanas de Guerrero y la Costa Oaxaqueña, en temas de derechos humanos, educación, salud, discriminación, racismo, xenofobia y violencia. Recuperado de http://www.cndh.org.mx/sites/all/doc/Comunicados/2016/ Com_2016_132.pdf.

Centro Centro de los Derechos Humanos Miguel Agustín Pro Juárez. (2015).

Exigen la aprobación de la reforma sobre los derechos de los pueblos indígenas y afromexicanos. Recuperado de http://centroprodh.org.mx/index. php?option=com_content\&view=article\&id=1669\%3A2015-10-13-17-42$12 \&$ catid $=186 \% 3$ Aeventos-pasados\&lang $=$ es

Consejo Nacional para Prevenir la Discriminación [Conapred]. (2010). Documento de respuesta sobre el trabajo de la conpared para eliminar la discriminación contra los descendientes de africanos y propuestas de acciones a realizarse con motivo del año internacional de los pueblos de descendencia africana. Recuperado de https:// www.conapred.org.mx/userfiles/files/Acciones_con_motivo_del_ao_ internacional_afros.pdf

Consejo Nacional para Prevenir la Discriminación [Conapred]. (2015). Otorga Asamblea Consultiva del Conapred, Premios por la Igualdad y la No Discriminación 2015. Consejo Nacional para Prevenir la Discriminación.

Recuperado de http://www.conapred.org.mx/movil_smartphone/index. php?contenido=noticias\&id=5700\&id_opcion=108\&op $=214$

Congreso de la Ciudad de México. (5 de febrero del 2017). Constitución Política de la Ciudad de México. 
Congreso del Estado Libre y Soberano de Guerrero. (2014). Constitución Política del Estado Libre y Soberano de Guerrero. Recuperado de https://www.gob. $\mathrm{mx} / \mathrm{cms} /$ uploads/attachment/file/173564/Constitucion_politica_estado_ libre_soberano_guerrero.pdf

Congreso del Estado Libre y Soberano de Oaxaca. (4 de abril de 1922) Constitución Política del Estado Libre y Soberano de Oaxaca.

Consejo para Prevenir y Eliminar la Discriminación de la Ciudad de México [Copred]. (2015). México sede Internacional en el reconocimiento de afrodescendientes. Copred. Recuperado de http://data.copred.cdmx.gob. $\mathrm{mx} /$ comunicacion-social-y-prensa/boletines/boletines-2015/mexico-sedeinternacional-en-el-reconocimiento-de-afrodescendientes/

E-Oaxaca. (2015). Reforma Constitucional, reconoce derechos de pueblos indigenas y afromexicanos: JBCG. Recuperado de http://www.e-oaxaca.com/nota/201507-26/congreso/reforma-constitucional-reconoce-derechos-de-pueblosindigenas-y

Espinosa-Bonilla, A. (2011). Activismo global: nuevas rutas de acción colectiva del movimiento negro en Colombia. Universitas Humanística, 2011(72), 211-245.

Esquivel, L. (2017). Iniciativa que reforma los artículos 2, 27, 28 y 155 de la Constitución Política de los Estados Unidos Mexicanos, a cargo de la Dip. Laura Beatriz Esquivel Valdes (Morena). Recuperado de http://sil.gobernacion.gob.mx/Archivos/ Documentos/2017/04/asun_3534670_20170427_1492452820.pdf

Escobar, A. (2010). Territorios de diferencia: Lugar, movimientos, vida, redes. Carolina del Norte: Envión Editores.

García, B. \& Benítez, R. (2013). Los movimientos afromexicanos en la Costa Chica. Recuperado de www.nacionmulticultural.unam.mx/ reconocimientopueblosnegros/docs/118.pdf.

García, J. (2017). Plantea PAN reconocer pueblos afromexicanos. Recuperado de https:// palabrasclaras.mx/estatal/plantea-pan-reconocer-pueblos-afromexicanos/

Gasga, E. (2013). Mujeres y poder político. En Conmemoración del 60 aniversario del voto femenino en México. Ciudad de México: Tribunal Electoral del Poder Judicial.

Girón, Y. (2015). Acción colectiva transnacional y lucha por los derechos humanos en el movimiento afrocolombiano (2002-2010). Ecuador: Flacso.

Hoffmann, O. \& Lara, G. (2012). Reivindicación afromexicana: formas de organización de la movilización negra en México. En M. Becerra, D. Buffa, H. Noufouri, y M. Ayala (eds.), Las poblaciones afrodescendientes de América latina y el Caribe. Pasado, presente y perspectivas desde el siglo XXI (pp. 25-46). Córdoba: Ciecs.

Htun, M. (2004). From "Racial Democracy" to affirmative action: changing state policy on race in Brazil. Latin American Research Review, 39(1), 60-98. Recuperado de http://lasa-4.univ.pitt.edu/LARR/prot/fulltext/vol39nol/ Htun.pdf

Instituto Nacional de Estadística y Geografía [Inegi]. (2017). Presentan publicación sobre perfil de la población afrodescendiente en México. Recuperado de http://www. 
inegi.org.mx/saladeprensa/boletines/2017/especiales/especiales2017_03_04. pdf

Instituto Nacional de Estadística y Geografía [Inegi]. (2015). Principales resultados de la Encuesta Intercensal 2015. Recuperado de http://internet.contenidos. inegi.org.mx/contenidos/productos/prod_serv/contenidos/espanol/ bvinegi/productos/nueva_estruc/702825078966.pdf.

Keck, M. \& Sikkink, K. (1998). Activists beyond Borders: Advocacy Networks in International Politics. Ithaca: Cornell University Press.

Keck, M. \& Sikkink, K. (1999). Transnational advocacy networks in international and regional politics. International Social Science Journal, 51(1), 89-101. Recuperado de https://onlinelibrary.wiley.com/doi/abs/10.1111/14682451.00179

Khagram, S., Riker, J., \& Sikkink, K. (2002). From Santiago to Seattle: Transnational Advocacy Groups Restructuring World Politics. En S. Khagram (ed.), Restructuring World Politics (pp. 3-23). Minnesota: University of Minnesota Press

Martí, S. \& Silva, E. (2014). Introducción: movilización y protesta en el mundo global e interconectado. Revista Cidob D'afers Internacionals, 105, 7-18. Recuperado de https://www.jstor.org/stable/24364564

Masferrer, C. (2017) Los retos de México en el Decenio Internacional para los Afrodescendientes (2015-2024). Dfensor, 7(15), 10-15. Recuperado de https://cdhdf.org.mx/wp-content/uploads/2014/05/dfensor_07_2017.pdf

Masferrer, C. \& Ramírez, S. (2016) ¿Por qué los afromexicanos en la constitución? El Universal. Recuperado de http://www.eluniversal.com.mx/entrada-deopinion/colaboracion/cristina-masferrer-leon-y-sergio-ramirez-caloca/ nacion/2016/07/17ñ

Miranda, M. (2012). Soy la negra de la costa: La reconfiguración de la identidad de género de mujeres afromexicanas de la Costa Chica. México: Flacso.

Nolan, K. (2015). Network Dynamics and Local Labor Rights Movements in Puebla, Mexico. En E. Silva (Ed.), Transnational Activism and National Movements in Latin America (pp. 106-140). Nueva York: Routledge.

Oxfam México. (2015a). ¿Qué pasó con mis derechos? Recuperado de http://atencion. oxfammexico.org/que-paso-con-mis-derechos/\#.We1OiDBrzIU

Oxfam México. (2015b). Pueblos indígenas combatiendo la discriminación. Recuperado de http://atencion.oxfammexico.org/nuevo-proyecto-de-oxfam-mexico-enoaxaca/\#.We1OsTBrzIV

Peñaloza-Pérez, P. S. (2017) La lucha por el reconocimiento de la población afrodescendiente en México (2015-2024). Dfensor, 7(15), 26-29. Recuperado de https://cdhdf.org.mx/wp-content/uploads/2014/05/dfensor_07_2017.pdf

Piden implementar políticas públicas a favor de afromexicanos. (2015a). 20 Minutos. Recuperado de http://www.20minutos.com.mx/noticia/b299231/ piden-implementar-politicas-publicas-a-favor-de-afromexicanos/

Quintana, R. \& Alanís, M. A. (2017) El reconocimiento de los derechos humanos de la población afrodescendiente en la Constitución Política de la Ciudad 
de México. Dfensor, 7(15), 32-37. Recuperado de https://www.jstor.org/ stable/24364564.

Reconocer los derechos de la población afromexicana, urge el Conapred.

(2017). 20 Minutos. Recuperado de https://www.20minutos.com.mx/ noticia/260797/0/reconocer-los-derechos-de-la-poblacion-afromexicanaurge-el-conapred/\#xtor=AD-1\&xts=513356

Reconocerá la nueva Constitución al pueblo afro como la quinta etnia de la entidad. (2012). El Sur. Recuperado de https://suracapulco.mx/archivoelsur/ $\operatorname{archivos} / 1547$.

Rojas, A. (2013). El surgimiento de lo afrodescendiente en América Latina y el Caribe. Textura, 27, 5-32. Recuperado de http://www.periodicos.ulbra.br/ index.php/txra/article/download/948/736.

Secretaría de Asuntos Indígenas. (2011). Documentos del Encuentro de Pueblos Indígenas de Oaxaca con la Alta Comisionada de la ONU para los Derechos Humanos Oaxaca. Recuperado de http://www.sai.oaxaca.gob.mx/wp-content/ uploads/2016/09/DE_ACDH.pdf

Secretaría de Cultura. (2017). Instauran la Cátedra Unesco Afrodescendientes en México y Centroamérica. Recuperado de http://www2.inah.gob.mx/es/boletines/6138instauran-la-catedra-unesco-afrodescendientes-en-mexico-y-centroamerica

Senado y Segob promueven reconocimiento constitucional de pueblos afromexicanos. (2015). mvs Noticias. Recuperado de http://www.mvsnoticias. com/\#!/noticias/senado-y-segob-promueven-reconocimiento-constitucionalde-pueblos-afromexicanos-611

Soto, A. (2015). Exigen se reconozca a las afromexicanas en la Constitución. Cimac Noticias. Recuperado de http://www.cimacnoticias.com.mx/node/70101.

Tarrow, S. (1997). El poder en movimiento: Los movimientos sociales, la acción colectiva y la politica. Madrid: Alianza.

Tarrow, S. (2005). The New Transnational Activism. Cambridge: Cambridge University Press.

De la Torre, V. (2011). La acción colectiva transnacional en la acción colectiva de los movimientos sociales y de las Relaciones Internacionales. confines de Relaciones Internacionales y Ciencia Política, 7(14), 45-72. Recuperado de https://confines.mty.itesm.mx/articulos14/Laaccioncolectiva.pdf

Valencia, L. (2015). Ambigüedades en dos décadas de paradigma multiculturalista. Algunos elementos de la historia inmediata de los Afrocolombianos. Revista cS, 16, 16-37. Recuperado de https://www.icesi.edu.co/revistas/index.php/ revista_cs/article/view/1967.

Valera, S. \& Pol, E. (1994). El concepto de identidad social urbana: una aproximación entre la psicología social y la psicología ambiental. Anuario de Psicología - The UB Journal of Psychology, 62, 5-24. Recuperado de https://www. raco.cat/index.php/AnuarioPsicologia/article/view/61126

Velázquez, M. (2017). Retos en los estudios afromexicanos. Congreso Internacional Estudios Afromexicanos: reflexiones, debates y retos. 5 de septiembre, Oaxaca, México. 
Velázquez, M. \& Iturralde, G. (2016). Afromexicanos. Reflexiones sobre las dinámicas del reconocimiento. Anales de Antropología, 50(2), 232-246.

Recuperado de http://www.revistas.unam.mx/index.php/antropologia/ article/download/56608/50232.

Velázquez, M. \& Iturralde, G. (2012). Afrodescendientes en México: Una historia de silencio y discriminación. Ciudad de México: Conapred. Recuperado de https:// www.conapred.org.mx/userfiles/files/TestimonioAFRO-INACCSS(1).pdf

Wade, P. (2000). Raza y etnicidad en Latinoamérica. Quito: Ediciones ABYA-YALA.

Wendt, A. (1992). Anarchy is what States Make of it: The Social Construction of Power Politics. International Organization, 46(2), 391-425. Recuperado de https://www.jstor.org/stable/2706858?seq=1/subjects.

Wright, C. (2018). El derecho a la consulta previa de los pueblos indígenas de México. Un balance de su reconocimiento, implementación e instrumentalización. En C. Wright (ed.), Participación política indígena en México. Experiencias de gestión comunitaria, participación institucional y consulta previa (pp. 219-256). Ciudad de México: Editorial Ithaca/Universidad de Monterrey.

Zehfuss, M. (2009). Constructivism in International Relations: The Politics of Reality. Cambridge: Cambridge University Press. 